\title{
Quitosano: una alternativa sustentable para el empaque de alimentos
}

\author{
César Eulogio Ortega Cardona y Xochitl Aparicio Fernández
}

\begin{abstract}
Resumen
Las películas y recubrimientos comestibles (PRC) se elaboran a partir de biopolímeros naturales y comestibles, como lípidos, polisacáridos y proteínas. Después de la celulosa, la quitina es uno de los biopolímeros más abundantes en la naturaleza, de la cual se obtiene el quitosano por un proceso de desacetilación, que consiste en la eliminación de grupos acetilo $\left(-\mathrm{CO}-\mathrm{CH}_{3}\right)$, generalmente por tratamientos con hidróxidos a altas temperaturas, y la formación de grupos amino $\left(\mathrm{NH}_{2}\right)$. El quitosano es altamente biodegradable y tiene propiedades de formación de películas, por lo que en años recientes ha sido bastante utilizado en el desarrollo de PRC, lo que representa una reducción importante en la contaminación ambiental. Su uso y aplicación se ha centrado en alimentos mínimamente procesados, con la característica de ser muy perecederos, como frutas, verduras, carnes, etcétera. Los últimos desarrollos involucran la adición de productos naturales que les otorga un carácter polifuncional, como antimicrobiano, antioxidante e, incluso, mejoran su calidad organoléptica y nutricional.
\end{abstract}

Palabras clave: quitosano, películas comestibles, recubrimientos comestibles, envasado activo, calidad de alimentos.

\section{Chitosan, a sustainable alternative fOR fOOd PACKAgING}

\begin{abstract}
Edible films and coatings are made from natural and edible biopolymers, such as lipids, polysaccharides, and proteins. After cellulose, chitin is one of the most abundant biopolymers in nature, from which chitosan is obtained by a deacetylation process, which consists in the elimination of acetyl groups (-CO$\left.\mathrm{CH}_{3}\right)$, generally by treatments with hydroxides at high temperatures, and the formation of amino groups $\left(\mathrm{NH}_{2}\right)$. Chitosan is highly biodegradable and has film-forming properties, so in recent years it has been widely used in the development of edible films and coatings, which represents a significant reduction in environmental pollution. Its use and application have focused mainly on highly perishable, minimally processed, foods such as fruits, vegetables, meats, etc. The latest developments involve the addition of natural products that give them a polyfunctional character, such as antimicrobial, antioxidant and even that improve their organoleptic and nutritional quality.
\end{abstract}

Keywords: chitosan, edible films, edible coatings, active packaging, food quality.

Recepción: 07/05/2019. Aceptación: 20/05/2020. Dol: http://doi.org/10.22201/cuaieed.16076079e.2020.21.5.4 


\section{César Eulogio Ortega Cardona}

ibicesarortega@gmail.com orcid.org/0000-0002-8433-5734

Actualmente es estudiante de la Maestría en Ciencia y Tecnología del Centro Universitario de los Lagos, Universidad de Guadalajara. Es Ingeniero Bioquímico por la Universidad de Guadalajara. Ha participado en varios proyectos de investigacion en el área de alimentos y tiene experiencia de 8 años en empresas de alimentos del sector privado. Sus intereses de investgación son la biotecnología y los productos naturales.

\section{Xochitl Aparicio Fernández}

xaparicio@culagos.udg.mx orcid.org/0000-0002-5980-085X

Profesora de Tiempo Completo en el Departamento de Ciencias de la Tierra y de la Vida del Centro Universitario de los Lagos, Universidad de Guadalajara. Es Doctora en Ciencias de los Alimentos por la Universidad Autónoma de Querétaro y, Química-Bióloga, con especialidad en Tecnología de Alimentos, por la Universidad de Sonora. Desarrolla investigación en el área de alimentos nutracéuticos y productos naturales de la región, así como en el aprovechamiento de subproductos alimenticios. Es miembro del Sistema Nacional de Investigadores, nivel I, cuenta con perfil PROMEP, es líder del Cuerpo Académico en formación "UDG-CA-1036 Biociencias en los productos naturales y sintéticos, y su impacto en la salud".

\section{Introducción}

La sustentabilidad ha impactado en casi en todos los sectores, y la industria de materiales de empaque no ha sido la excepción, motivada principalmente por la exigencia y necesidad de los consumidores de preservar y cuidar el medio ambiente. En la búsqueda para reducir el impacto generado por la fabricación, uso y disposición de los materiales plásticos derivados del petróleo, el desarrollo de películas y recubrimientos comestibles (PRC) ha surgido como respuesta para satisfacer esta necesidad. Algunas de las funciones que deben cumplir dichas PRC son: proteger el producto de daños mecánicos, físicos, químicos y microbiológicos; y mantener su calidad sensorial y comercial por más tiempo. Su uso se ha centrado en alimentos altamente perecederos y/o mínimamente procesados.

La principal ventaja de las PRC sobre los empaques sintéticos tradicionales es que pueden ser consumidas con los productos envasados, e, incluso si las películas no se consumieran, no representan un problema de contaminación ambiental. Todos los alimentos, especialmente las frutas, hortalizas y carnes, son susceptibles a la descomposición; cada año hasta un tercio de ellos se estropea (Food and Agriculture Organization of the United Nations [FAO], 2012), lo que representa grandes pérdidas económicas para la industria alimentaria. El desarrollo de PRC a base de quitosano se considera una alternativa práctica y eficiente para disminuir el deterioro de los alimentos. El objetivo de la presente revisión es difundir la información sobre la producción, aplicaciones y beneficios de las PRc basadas en quitosano, debido a sus características de buena formación de película, su carácter no tóxico, su alta biodegradabilidad, sus propiedades mecánicas y de barrera para la protección del alimento y su carácter antimicrobiano (Chillo et al., 2008), 
Imagen 1. Estructura de la quitina y quitosano. Fuente: elaboración propia, basado en Muxika et al., 2017.

Imagen 2. Funciones de las películas y recubrimientos comestibles.

\section{Quitina y quitosano}

La quitina es uno de los biopolímeros naturales más abundantes, se encuentra como componente estructural en el exoesqueleto de los crustáceos, en paredes celulares de hongos y en otros materiales biológicos; su abundancia y carácter natural lo hacen muy atractivo, por lo que ha sido objeto de varios estudios científicos. Cada año, se producen 100 billones de toneladas de quitina por moluscos, crustáceos, insectos, hongos y algas (Muxika et al., 2017). Los residuos de conchas de crustáceos son una fuente importante de contaminación en áreas costeras y representan una alternativa económicamente viable para la producción de quitosano, especialmente si se incluye la recuperación de carotenoides (Dutta et al., 2004). Su estructura química consiste en una cadena lineal de unidades $\mathrm{N}$-acetil-D-glucosamina unidas por enlaces glicosídicos $\beta$-1,4; por sus características químicas es insoluble en todos los solventes comunes, incluyendo el agua y solventes orgánicos (ver imagen 1).
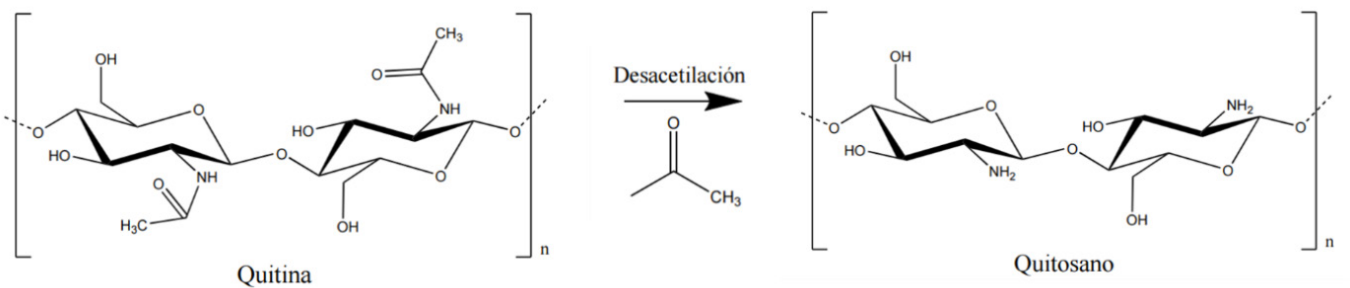

Una reacción de desacetilación alcalina al 50, 60\% o más convierte a la quitina en quitosano. Esta reacción consiste en la eliminación del grupo acetilo de la cadena lineal y la formación de un grupo amino, que origina dos unidades distintas: la D-glucosamina y N-acetil-D-glucosamina. Dicha modificación en la estructura genera un compuesto soluble en soluciones acuosas ácidas. El quitosano es biodegradable, no tóxico, tiene propiedades antimicrobianas (antibacterial y antifúngico), antioxidantes y está altamente disponible de manera comercial. El quitosano tiene varias aplicaciones en el tratamiento de aguas residuales, agricultura, biomedicina, industria farmacéutica, cosmética, alimentaria, entre otras.

\section{Películas y recubrimientos comestibles}

El envasado activo de alimentos consiste en la incorporación de compuestos activos (antimicrobianos, antioxidantes) en los materiales 
de envasado, que generalmente se utilizan como películas o recubrimientos. Se denomina recubrimiento comestible (RC) a la capa delgada de material que se coloca sobre un alimento y es seguro para ser consumido. La principal diferencia entre películasy recubrimientos comestibles se basa en su forma de aplicación; las películas comestibles (PC) se forman por separado del alimento para posteriormente ser utilizadas para recubrir el mismo, mientras que los Rc se forman directamente sobre la superficie del alimento. Ambos se elaboran a partir de polímeros comestibles como componente principal, tales como lípidos, polisacáridos y proteínas (Montalvo et al., 2012); cumplen funciones similares a las de los envases sintéticos convencionales, al proteger el producto de daños mecánicos, físicos, químicos y microbiológicos; y actúan como barreras contra el vapor de agua y gases, mejoran la integridad estructural y las propiedades de manipulación mecánica de los alimentos (Cordeiro de Azeredo, 2012, ver imagen 2).

Su uso en aplicaciones alimentarias y especialmente en productos altamente perecederos (frutas, hortalizasy carnes) se basa en algunas propiedades particulares, tales como bajo costo, disponibilidad, atributos funcionales, propiedades mecánicas (flexibilidad, tensión), propiedades ópticas (brillo y opacidad), reducción de transporte de gases $\left(\mathrm{CO}_{\text {y }} \mathrm{CO}_{2}\right)$, resistencia estructural al agua y microorganismos, y su aceptabilidad sensorial (Falguera et al., 2011).

\section{Elaboración de películas y recubrimientos comestibles}

Para la creación de PC y Rc se requieren los siguientes componentes: un biopolímero formador de película, un plastificante y, opcionalmente, aditivos; éstos se agregan a un disolvente adecuado para preparar la suspensión formadora de película (sFp, ver imagen 3).

Imagen 3. Composición de las películas y recubrimientos comestibles.

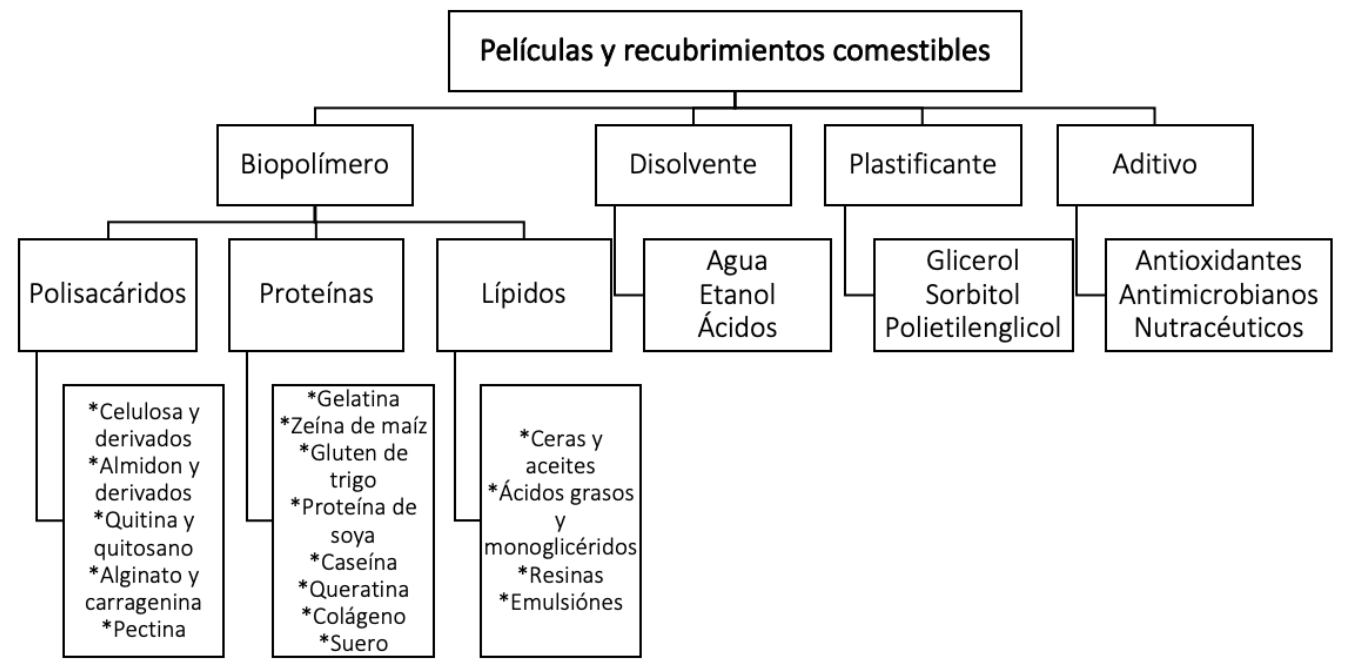

LaS PRC pueden ser desarrollados a partir de un solo biopolímero estructural o de una mezcla de ellos, los cuales forman películas por múltiples mecanismos, incluyendo enlaces covalentes, interacciones electrostáticas, hidrofóbicas, iónicas 
y puentes de hidrógeno (Suput y Popović, 2015). La estructura continua formada puede ser cristalina o amorfa, y actúa a manera de barrera para proteger al alimento (Cruz-Morfin et al., 2013) y prolongar su vida útil.

Las PRC basadas en polisacáridos tienen una buena resistencia mecánica, moderadas propiedades de barrera a los gases, son comestibles y fácilmente degradables, sin embargo, son muy frágiles e hidrofílicas (da Rocha et al., 2018). Las PRc basadas en proteínas son hidrófilas por naturaleza por lo que ofrecen poca resistencia al agua y poca resistencia mecánica, lo que limita su aplicación en el envasado de alimentos, sus propiedades se ven influenciadas principalmente por la composición y estructura de la proteína. Las PRC elaboradas a base de lípidos tienen propiedades de barrera contra el oxígeno limitadas; sin embargo, presentan buenas propiedades de barrera contra el vapor de agua y son poco flexibles (Ciolacu et al., 2013); por su naturaleza hidrofóbica generalmente se formulan en combinación con polisacáridos y proteínas. Los últimos desarrollos en PRC combinan estos biopolímeros estructurales con la inclusión de aditivos alimentarios de carácter natural, para mejorar su funcionalidad y propiedades y aporten algún beneficio al ser consumidos.

El mecanismo de formación de películas basadas en polisacáridos es el rompimiento del polímero en segmentos y la regeneración de la cadena del polímero al interior de la matriz de la película. Esto es usualmente logrado por evaporación del solvente, creando enlaces hidrofílicos con hidrógeno, enlaces iónicos y/o reticulación electrolítica e iónica (Butler et al., 1996). Las cadenas se asocian entre sí por enlaces de hidrógeno muy fuertes entre los grupos amida y grupos carbonilo de las cadenas cercanas; lo que además también les permite interactuar con otras moléculas adicionadas (Kardas et al., 2012).

Las tecnologías existentes para la aplicación de las PRc se basan en los métodos de moldeo, recubrimiento e inmersión (casting, coating, dipping, en inglés). El moldeo se emplea para la formación de PC; la sfp se vierte en un molde y es sometida a un proceso de secado que da origen a la pc propiamente dicha (ver figura 4).

Imagen 4. Ejemplo de películas comestibles a base de quitosano.



Los métodos de recubrimiento e inmersión originan Rc. En el método de recubrimiento, la SFP se aplica directamente en la superficie de los alimentos con apoyo de herramientas, tales como brochas, espátulas o rociadores; mientras que en el método de inmersión el alimento se sumerge en la sFP (ver imagen 5). En ambos casos el alimento recubierto se somete a secado para lograr la formación de la pc en la superficie del alimento (ver imagen 6). La formación de una buena PRC depende en 


\section{Conclusión}

La buena capacidad de formación de película del quitosano, su carácter biodegradable, antimicrobiano y su versatilidad para la combinación con aditivos, principalmente de carácter natural, lo convierten en un elemento central para investigaciones en el desarrollo de empaques sustentables y funcionales. Las PRC a base de quitosano han demostrado tener buenas propiedades mecánicas y de barrera que pueden ayudar a mantener la vida útil y mejorar la calidad nutrimental de alimentos mínimamente procesados. La adición de componentes de origen natural puede mejorar las propiedades específicas de las PRC de quitosano. Es importante continuar con la investigación en la adición de elementos naturales para mejorar las propiedades y fomentar el uso de PRC a base de quitosano en la industria de alimentos.

\section{Referencias}

* Asik, E., y Candogan, K. (2014). Effects of chitosan coatings incorporated with garlic oil on quality characteristics of shrimp. Journal of Food Quality, 37(4), 237-246. DOI: https://doi.org/10.1111/jfq.12088

* Butler, B. L., Vergano, P.J., Testin, R. F., Bunn, J. M.,y Wiles, J. L. (1996). Mechanical and barrier properties of edible chitosan films as affected by composition and storage. Journal of Food Science, 61(5), 953-956. Dol: https://doi.org/10.1111/j.1365-2621.1996. tb10909.x

* Chillo, S., Flores, S., Mastromatteo, M., Conte, A., Gerschenson, L., y Nobile, M. A. Del. (2008, septiembre). Influence of glycerol and chitosan on tapioca starchbased edible film properties. Journal of Food Engineering, 88(2), 159-168. Dol: https://doi.org/10.1016/j.jfoodeng.2008.02.002

- Ciolacu, L., Nicolau, A. I., y Hoorfar, J. (2014). 17 - Edible coatings for fresh and minimally processed fruits and vegetables. En Global Safety of Fresh Produce: A Handbook of Best Practice, Innovative Commercial Solutions and Case Studies (pp. 233244). Woodhead Publishing. Dol: https://doi.org/10.1533/9781782420279.3.233

Cordeiro de Azeredo, H. M. (2012). 14 - Edible coatings. Advances in Fruit Processing Technologies. CRC Press Inc.

* Cruz Morfin, R., Martínez Tenorio, R., y López Malo Vigil, A. (2013). Biopolímeros y su integración con polímeros convencionales como alternativa de empaque de alimentos. Temas de Selección de Ingeniería de Alimentos, 7(2), 42-52.

* da Rocha, M., de Souza, M. M., y Prentice, C. (2018). Chapter 9 - Biodegradable films: An alternative food packaging. En Food Packaging and Preservation (pp. 307-342). Elsevier Inc. Dol: https://doi.org/10.1016/b978-0-12-811516-9.00009-9

* Dutta, P. K., Dutta, J., y Tripathi, V. S. (2004, enero). Chitin and Chitosan: Chemistry, properties and applications. Journal of Scientific and Industrial Research, 63(1), 20-31. https://pdfs.semanticscholar.org/af57/fa5a2b237174301b9b2740adbbe531a4a527. pdf?_ga=2.211638044.865596640.1596761652-451637070.1596674569 
* Falguera, V., Quintero, J. P., Jiménez, A., Muñoz, J. A., y Ibarz, A. (2011, 31 de mayo). Edible films and coatings: Structures, active functions and trends in their use. Trends in Food Science y Technology, 22(6), 292-303. Dol: https://doi.org/10.1016/j. tifs.2011.02.004

* Fathima, P. E., Panda, S. K., Ashraf, P. M., Varghese, T. O., y Bindu, J. (2018, 1 de octubre). Polylactic acid/chitosan films for packaging of Indian white prawn (Fenneropenaeus indicus). International Journal of Biological Macromolecules, 117, 1002-1010. Dol: https://doi.org/10.1016/j.jibiomac.2018.05.214

* Food and Agriculture Organization of the United Nations [FAo]. (2012). Pérdidas y desperdicio de alimentos en el mundo - Alcance, causas y prevención. http://www.fao. org/3/a-i2697s.pdf

- Genskowsky, E., Puente, L. A., Pérez-Álvarez, J. A., Fernandez-Lopez, J., Muñoz, L. A., y Viuda-Martos, M. (2015, diciembre). Assessment of antibacterial and antioxidant properties of chitosan edible films incorporated with maqui berry (Aristotelia chilensis). LWT - Food Science and Technology, 64(2), 1057-1062. Dol: https://doi.org/10.1016/j.Iwt.2015.07.026

* Gol, N. B., Chaudhari, M. L., y Rao, T. V. R. (2015). Effect of edible coatings on quality and shelf life of carambola (Averrhoa carambola L.) fruit during storage. Journal of Food Science and Technology, 52, 78-91. Dol: https://doi.org/10.1007/s13197-013-0988-9

* Kardas, I., Struszczyk, M. H., Kucharska, M., van den Broek, L. A. M., van Dam, J. E. G., y Ciechańska, D. (2012). Chitin and Chitosan as Functional Biopolymers for Industrial Applications. En P. Navard (Ed.), The European Polysaccharide Network of Excellence (EPNOE) (pp. 329-373). Springer Vienna.

* Kurek, M., Garofulić, I. E., Bakić, M. T., Ščetar, M., Uzelac, V. D., y Galić, K. (2018, noviembre). Development and evaluation of a novel antioxidant and $\mathrm{pH}$ indicator film based on chitosan and food waste sources of antioxidants. Food Hydrocolloids, 84, 238-246. Dol: https://doi.org/10.1016/j.foodhyd.2018.05.050

- Leceta, I., Peñalba, M., Arana, P., Guerrero, P., y de la Caba, K. (2015, mayo). Ageing of chitosan films: Effect of storage time on structure and optical, barrier and mechanical properties. European Polymer Journal, 66, 170-179. Dol: https://doi. org/10.1016/j.eurpolymj.2015.02.015

* Montalvo, C., López Malo, A., y Palou, E. (2012). Películas comestibles de proteína: características, propiedades y aplicaciones. Temas Selectos de Ingeniería de Alimentos, 6-2, 32-46. https://tsia.udlap.mx/peliculas-comestibles-de-proteinacaracteristicas-propiedades-y-aplicaciones-2/

- Muxika, A., Etxabide, A., Uranga, J., Guerrero, P., y de la Caba, K. (2017). Chitosan as a bioactive polymer: Processing, properties and applications. International Journal of Biological Macromolecules, 105, parte 2, 1358-1368. Dol: https://doi.org/10.1016/j. ijbiomac.2017.07.087

* Nair, M. S., Saxena, A., y Kaur, C. (2018). Effect of chitosan and alginate based coatings enriched with pomegranate peel extract to extend the postharvest quality of guava (Psidium guajava L.). Food Chemistry, 240, 245-252. Dol: https://10.0.3.248/j. foodchem.2017.07.122 
* Ojagh, S. M., Rezaei, M., Razavi, S. H., y Hosseini, S. M. H. (2010, 1 de septiembre). Development and evaluation of a novel biodegradable film made from chitosan and cinnamon essential oil with low affinity toward water. Food Chemistry, 122(1), 161-166. Dol: https://doi.org/10.1016/j.foodchem.2010.02.033

Suput, D., y Popović, S. (2015). Edible films and coatings: Sources, properties and application. Food and Feed Research, 42(1), 11-22. Dol: https://doi.org/10.5937/ FFR1501011S

* Wang, H., Qian, J., y Ding, F. (2018). Emerging Chitosan-Based Films for Food Packaging Applications. Journal of Agricultural and Food Chemistry, 66(2), 395-413. Dol: https://doi.org/10.1021/acs.jafc.7b04528

\section{Cómo CITAR ESTE ARTículo}

* Ortega Cardona, César Eulogio y Aparicio Fernández, Xochitl. (2020, septiembreoctubre). Quitosano: una alternativa sustentable para el empaque de alimentos. Revista Digital Universitaria (RDU), 21(5). Dol: http://doi.org/10.22201/ cuaieed.16076079e.2020.21.5.4 